\title{
Two rare missense mutations in the fibrillin-1 gene associated with atypical cardiovascular manifestations in a Chinese patient affected by Marfan syndrome
}

\author{
MIAO ZHANG, YAQI ZHOU, YANG PENG and LIJUN JIN \\ Department of Cardiology, The First Affiliated Hospital of Yangtze University, Jingzhou, Hubei 434000, P.R. China
}

Received April 5, 2016; Accepted March 21, 2017

DOI: $10.3892 / \mathrm{mmr} .2018 .9041$

\begin{abstract}
The present report aimed to evaluate the results of screen mutations of the fibrillin (FBN) 1 gene and analyze the symptoms in one Chinese patient clinically diagnosed with Marfan syndrome (MFS). Clinical data were collected and FBN1 gene sequencing was performed. Genomic DNA was extracted from the blood sample of the patient. All 65 exons were screened using a polymerase chain reaction assay. The diagnosis of MFS was confirmed via identification of symptoms presenting in the skeletal system (arachnodactyly, walker wrist and thumb signs) and the ocular system (ectopia lentis), in addition to a positive family history. The patient's cardiovascular manifestations (dilatation of the four cardiac chambers, severe mitral valve regurgitation and a large saccular aneurysm of the non-coronary sinus of Valsalva) were atypical to those that most frequently occur in cases of MFS. Following gene sequencing, two novel heterozygous mutations of the FBN-1 gene were identified: c.3442C $>\mathrm{G}$ in exon 27 , proline replaced with alanine (p. Pro1148Ala) and c.6388G $>$ A in exon 52, glutamic acid replaced with lysine (p. Glu2130Lys). The clinical symptoms and family history were important in the diagnosis of MFS, however the atypical signs that presented in the cardiovascular system may be associated with the disease, and may be noted for further cases in the future. Gene sequencing further verified the correct diagnosis of MFS.
\end{abstract}

\section{Introduction}

Marfan syndrome (MFS) is an autosomal dominant systemic disorder of connective tissue (1), which is characterized by defects in multiple organ systems, particularly the skeleton, eyes, heart and aorta. The incidence of MFS is $\sim 1 / 5,000-1 / 10,000$ (2). The fibrillin (FBN) 1 gene has been

Correspondence to: Dr Lijun Jin, Department of Cardiology, The First Affiliated Hospital of Yangtze University, 8 Hangkong Road, Jingzhou, Hubei 434000, P.R. China

E-mail: 305001136@qq.com

Key words: Marfan syndrome, mitral regurgitation, fibrillin 1, missense mutations identified as the primary disease-associated gene of MFS. A total of $\sim 3,000$ mutations, including all types of mutations in the FBN-1 gene, have been documented in the Universal Mutation Database (3). The FBN-1 gene contains 65 exons encoding a secreted $350 \mathrm{kDa}$ glycoprotein which is primarily composed of 3 types of repeated modules, including a cysteine-rich epidermal growth factor (EGF)-like domain, transforming growth factor $\beta-1$ binding protein-like module and a hybrid module (4). Furthermore, missense mutations are the most frequently occurring and account for $60 \%$ of overall mutations. Calcium binding (cb) via cbEGF modules has been hypothesized to be important in the conformation and stability of FBN-1 and the majority of the missense mutations that occur are located in the cbEGF modules (3).

Affected individuals of MFS have been identified to be tall and slender, and symptoms include arachnodactyly, scoliosis, and either a pectus excavatum, pectus carinatum, or ectopia lentis in the eyes (5). The predominant factors determining survival are the cardiovascular complications, which have been noted to be the primary cause of morbidity and mortality in MFS patients. The degree of the skeletal deformity has not been demonstrated to be associated with the severity and extent of the cardiovascular symptoms (6). The cardiovascular complications that are primarily associated with mortality include aortic root aneurysm and dissection, mitral valve regurgitation, calcification of the mitral valve annulus, dilatation or dissection of the descending aorta and mitral valve prolapse (7). Mitral valve alterations are the earliest manifestations and as they result in severe valve failure, are the primary causes of mortality in children (8). Aortic valve dysfunction occurs during the later stages of the disease progression and may be secondary to aortic root enlargement.

The present case study describes the atypical cardiovascular manifestations in a Chinese patient affected with Marfan syndrome. Symptoms included dilatation of the four cardiac chambers; however, the typical cardiovascular findings usually present, including dilatation or dissections of the ascending aorta, and mitral valve prolapse, were not present. Notably, echocardiography revealed severe mitral valve regurgitation, a large non-coronary sinus of Valsalva aneurysm and a tricuspid septal leaflet cleft. This was the first time the patient had heart failure symptoms without a typical aortic root enlargement and other classical changes, which were common in patients with MFS. Furthermore, 
gene-sequencing technology was used to analyze and verify the disease diagnosis. A total of two missense mutations in exon 27 and exon 52 of the FBN-1 gene were identified in the patient. These two rare mutations confirmed the diagnosis of the patient and to the best of our knowledge this is the first time atypical cardiovascular manifestations in patient with MFS have been reported.

\section{Case report}

Clinical evaluation. A 26-year-old male was admitted to the First Affiliated Hospital of Yangtze University (Jingzhou, China), with symptoms of palpitation, nausea, diarrhea and vomiting for two days. Physical examination revealed long limbs, (Fig. 1A and B) and myopia. Vital signs at presentation were blood pressure 110/70 $\mathrm{mmHg}$, heart rate $142 \mathrm{bpm}$ with irregular rhythm and respiratory rate $25 / \mathrm{min}$ and jugular venous distention was evident. Precordial movement with visible and palpable apical pulse at the 7th left intercostal space at the mid-axillary line was identified. On auscultation, inspiratory crackles at the two lung bases, irregular heartbeats and grade III/IV systolic murmur were audible over the entire precordial area, however prominent at the apex with thrill. Tricuspid or pulmonary auscultation was unable to be identified due to the rapid irregular heartbeats.

Ocular examination revealed dislocation of the lens (Fig. 2A) and diagnostic examination, including a chest X-ray, revealed a cardiothoracic index of $80 \%$ (Fig. 2B). An electrocardiogram revealed atrial fibrillation and positive criteria for biventricular dilatation. The diameters of the aortic root and the ascending aorta were 2.7 and $2.6 \mathrm{~cm}$ respectively, and a large non-coronary sinus of Valsalva aneurysm was detected via echocardiography, $5.27 \times 4.45 \mathrm{~cm}$ in size (Fig. 3A). A transthoracic echocardiogram revealed dilatation of the four cardiac chambers. Left ventricular end diastolic (LVEDD) and left ventricular end systolic (LVESD) dimensions were 9.83 and $7.70 \mathrm{~cm}$, respectively (Fig. 3B). The whole heart was severely dilated and hypokinetic. The left ventricular ejection fraction was $35 \%$. The dimensions in the right atrium, right ventricle and left atrium were 5.90, 3.10 and $7.80 \mathrm{~cm}$, respectively. Mitral prolapse, abnormal thickening or calcification of the mitral valve were not present, however, the degree of mitral regurgitation was severe (Fig. 3C). Mild tricuspid regurgitation and aortic regurgitation was present. In addition, the tricuspid septal leaflet cleft was observed (Fig. 3D).

The clinical features were evaluated according to the Ghent criteria and the first diagnosis of Marfan syndrome in the patient was verified. The therapeutic treatment administered to the patient was in consideration with the heart failure manifestations, and included intravenous diuretics, vasodilators and nasal oxygen, which resulted in symptomatic relief. The clinical condition of the patient was stabilized during the in-hospital stay. The valvular functioning and aortic diameter were regularly monitored and early administration of long-term-adrenergic blockade was prescribed to reduce hemodynamic stress on the proximal aorta, following hospital discharge. The patient followed up in the present department every 2 weeks and exacerbation of heart failure recently occurred 3 months after discharge from hospital due to a respiratory infection.
Family history. The proband, a 26-year-old male, was diagnosed with MFS according to the Ghent criteria (9). The family history revealed three affected family members over three generations, two of whom were deceased (Fig. 4). According to the description from the proband's uncle, all affected members had similar skeletal system manifestations, including tall stature, long limbs, arachnodactyly and decreased quality of vision.

Mutational analysis. A total of $2 \mathrm{ml}$ peripheral venous blood samples were obtained from the patient. All 65 FBN-1 exons and associated splice-junctions were amplified using the primers and conditions described by Nijbroek et al (10). Polymerase chain reaction (PCR) was conducted using a $30 \mu \mathrm{l}$ PCR amplification reaction mixture that contained $30 \mathrm{ng}$ genomic DNA, $1.0 \mu \mathrm{M}$ of each of the forward and reverse primers, and $15 \mu \mathrm{l}$ of 2x TaqMaster Mix (SinoBio Biotech Co. Ltd., Shanghai, China). Sequence data were compared with the FBN-1 sequences from the Human Genome database (GenBank NC_000015.10). A total of two heterozygous missense mutations, c.3442C $>\mathrm{G}$ in exon 27 and c.6388G $>\mathrm{A}$ in exon 52 were identified (Fig. 5). These heterozygous mutations resulted in the replacement of proline with alanine (p.Pro1148Ala) and glutamic acid with lysine (p.Glu2130Lys).

\section{Discussion}

MFS is a rare hereditary connective tissue disorder that affects numerous parts of the body. The age-associated nature of various clinical manifestations and variable phenotypic expression may complicate diagnosis. Currently, the diagnosis of MFS is primarily based on the Ghent criteria, which involves comprehensive evaluation of major and minor systemic manifestations. Major and minor criteria have been defined, based on family history, clinical manifestations in the various systems and genetic and molecular analysis. The clinical manifestations of MFS are pleiotropic and highly variable, with the affected systems involved to different degrees. MFS typically involves cardiovascular, musculoskeletal and ocular systems. According to the aforementioned criteria, a diagnosis of MFS requires one first-degree relative affected and involvement of two systems with major signs, or in the absence of a family history, the involvement of three systems, with major signs in at least two (9). In the present case study, skeletal and ocular systems were affected, with major signs in the skeletal (arachnodactyly, walker wrist and thumb signs) and the ocular systems (ectopia lentis) as previously revealed. Therefore, with the positive family history, the criteria for the diagnosis of MFS in the patient were completely fulfilled.

Skeletal manifestations are the most frequently occurring symptoms of MFS and usually gain the attention of physician. However, cardiovascular manifestations are the most serious complications and determine the prognosis and survival in MFS. Aortic root dilation and mitral valve prolapse are prominent clinical findings in patients with the disease (11), and have been recognized to be as prevalent as ocular defects (12). Sisk et al (13) revealed the presence of aortic root dilation and mitral valve prolapse in all 13 cases of MFS, which presented at ages of $<4$ years. The results of the present study are from the patient's first visit to a clinician, 
A
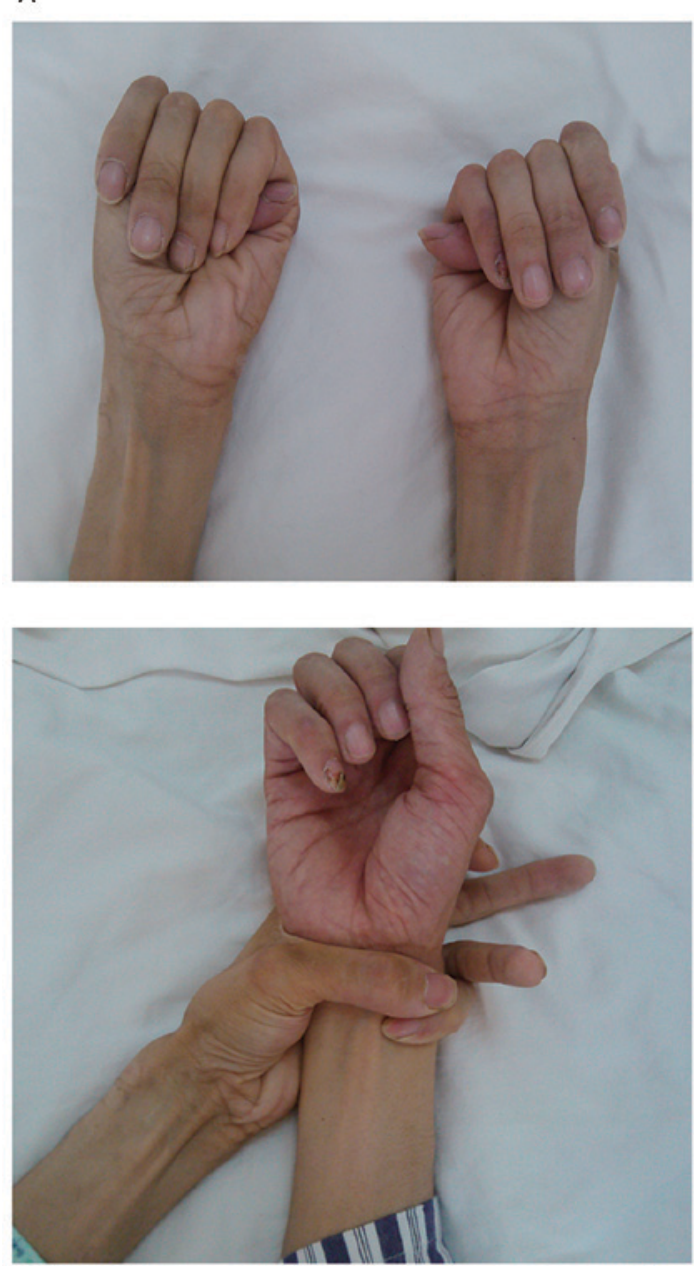

B
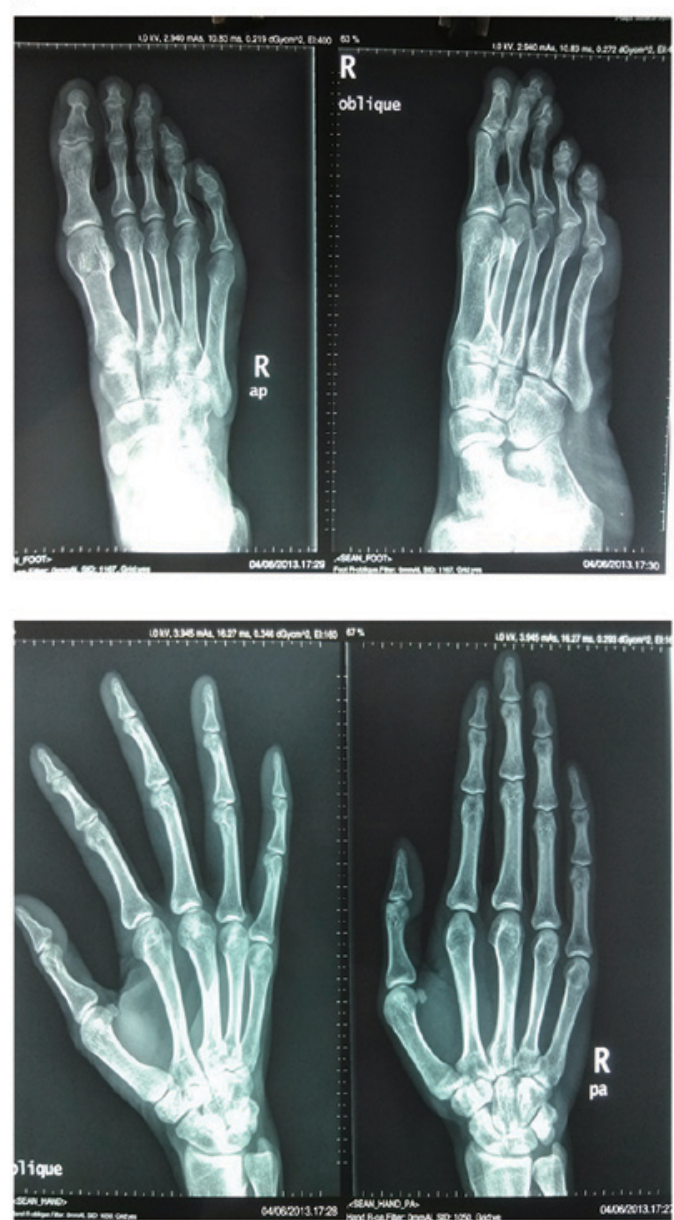

Figure 1. (A) Thumb sign and Walker-Murdoch wrist sign, used to determine arachnodactyly. (B) Dolichostenomelia.

A
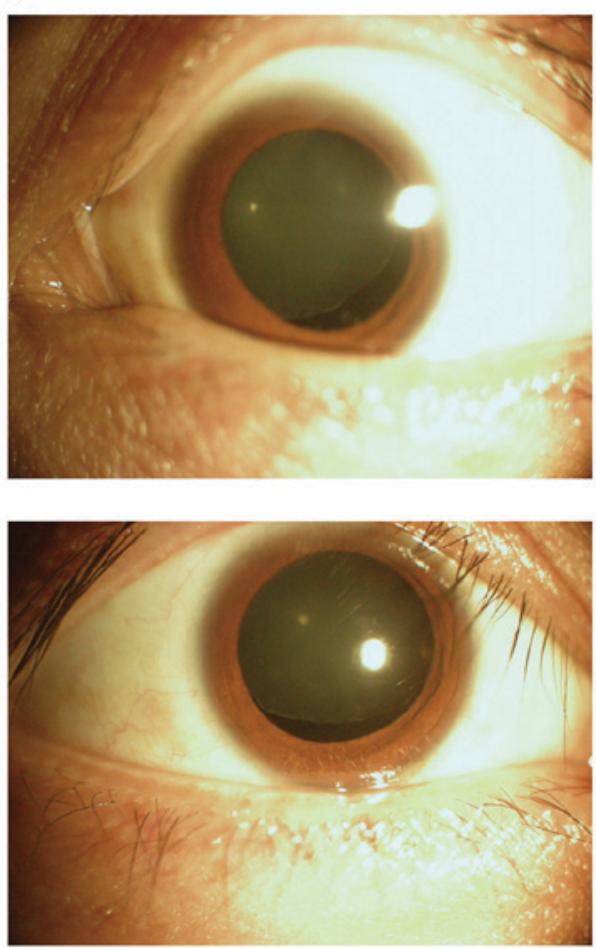

B

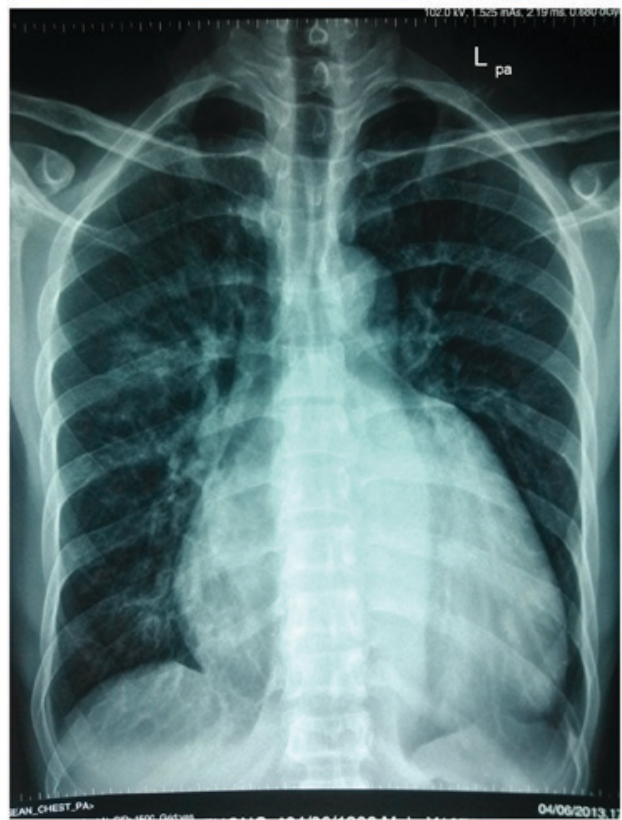

Figure 2. (A) Ectopic lens. (B) Chest X-ray. 
A

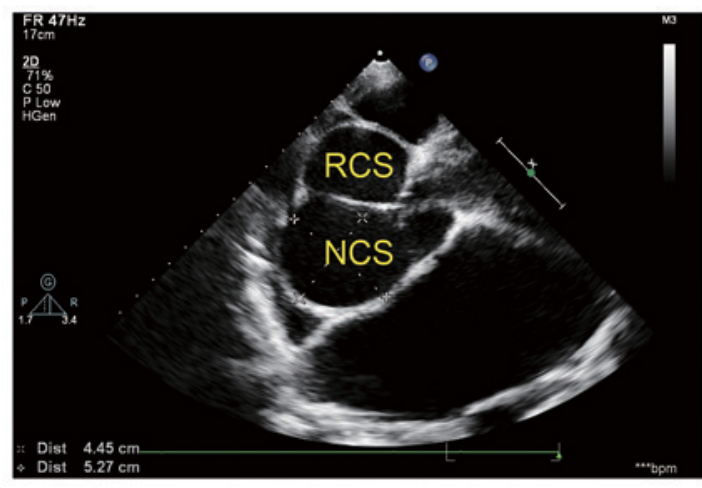

C

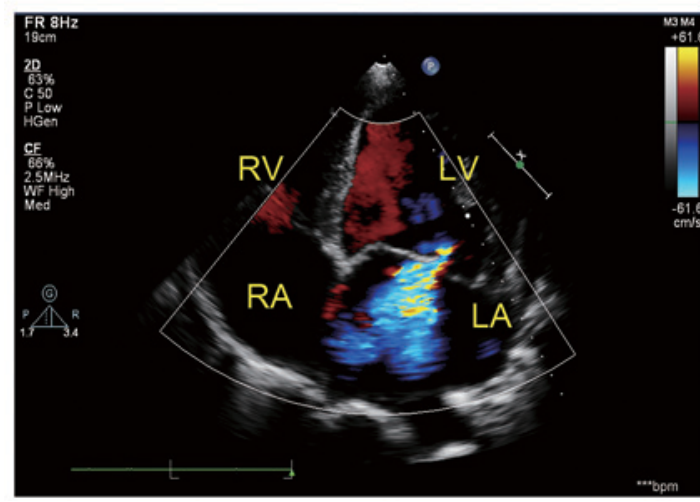

B

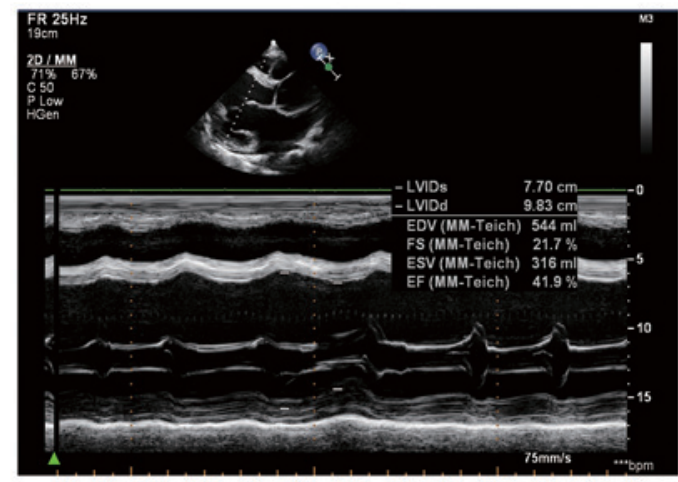

D

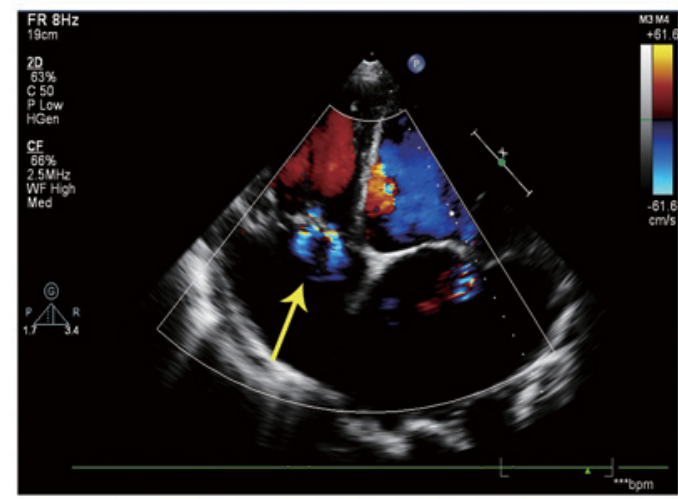

Figure 3. (A) Large NCS of Valsava aneurysm, $5.27 \times 4.45 \mathrm{~cm}$ in size. (B) M-mode transthoracic echocardiogram demonstrating left ventricular dysfunction with an ejection function of $41.9 \%$ and end diastolic and end systolic diameters of 9.83 and $7.7 \mathrm{~cm}$, respectively. (C) Color doppler showing severe mitral regurgitation. (D) Color Doppler showing tricuspid septal leaflet cleft, indicated with an arrow. NCS, noncoronary sinus; RCS, right coronary sinus; LA, left atrium; LV, left ventricle; RA, right atrium; RV, right ventricle.

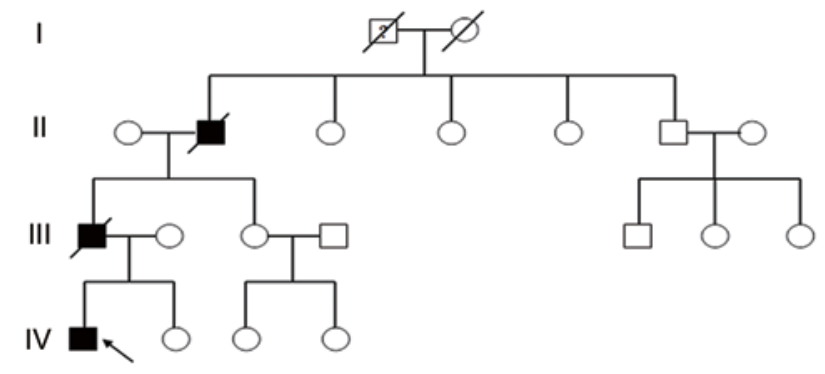

Figure 4. Pedigree of the family. Squares indicate males and circles indicate females. Filled-in symbols represent affected members. Crossed-out symbols denote that the subject is deceased. Symbols with a question mark in the center indicate that the member was not diagnosed clearly. Proband patient is indicated by an arrow.

and following examination, severe damage to the heart was revealed and a large saccular aneurysm of the non-coronary sinus was observed, without the dilatation or dissection of the ascending aorta. In addition to this, echocardiography revealed dilation of the four cardiac chambers and globally hypokinetic movement. The left ventricular ejection fraction was 35\%, with LVEDD and LVESD dimensions of 9.83 and $7.70 \mathrm{~cm}$, respectively. During the in-hospital stay, reversal of atrial fibrillation was achieved following the relieved symptoms of heart failure. The electrocardiogram examination of this patient appeared normal one year ago, so there was an important clue that the potential duration of atrial fibrillation was not long in this

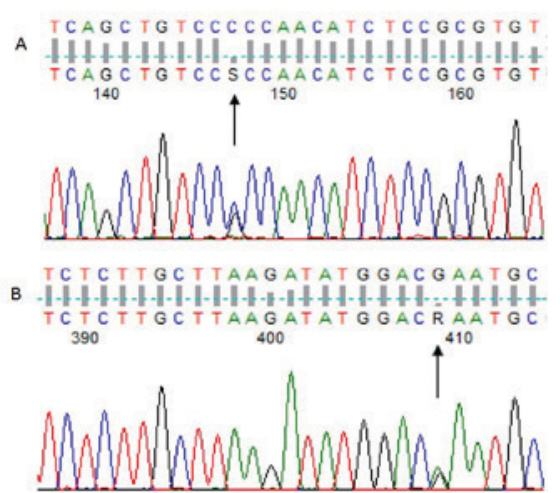

Figure 5. Sequencing results of the FBN1 gene. (A) Heterozygous missense mutation c.3442C $>\mathrm{G}$ in exon 27 (black arrow). (B) Heterozygous missense mutation c.6388G $>\mathrm{A}$ in exon 52 (black arrow).

patient, which indicated that the tachycardia should not be a dominating contributor to his myocardial dysfunction and the patient had not been suffering from atrial fibrillation for a long period. However, without prior serial imaging studies, a previous evaluation of the echocardiogram was unable to be verified.

A large saccular aneurysm of the non-coronary sinus of Valsalva without dilatation of the ascending aorta, and severe mitral valve regurgitation without mitral valve prolapse, were notable symptoms in the patient. The aim of the study was to 
assess the presence of any genetic mutations in FBN-1 associated with the unusual cardiovascular manifestations in the patient, and contribute to the molecular research regarding physical manifestations of MFS. A total of two missense mutations were identified in exon 27 (c.3442C $>$ G) and exon 52 (c.6388G $>$ A) in the FBN-1 sequence. Comeglio et al (14) reported a mutation in exon 27 (c.3442C $>$ G) of FBN-1 in a 3 -year-old British patient affected by MFS, which resulted in a proline to alanine substitution (P1148A) in cbEGF-like domains and the primary cardiovascular manifestation was aortic dilatation. Additionally, a mutation in exon52 (c.6388G $>$ A) of FBN-1 has been reported to result in mitral valve prolapse or aortic dilatation in a 15 -year-old woman (15). These two mutations are rare and appear separately in different patients. However, the two mutations occurred simultaneously in the current patient, who presented with differing cardiovascular manifestations compared with previous reports. However, all the cardiovascular symptoms are unable to be attributed to the two gene mutations exclusively, and further research is required in order to fully elucidate the molecular basis of the occurrence of the cardiovascular symptoms in this disease.

The patient presented with poor cardiac function and atypical cardiology symptoms, however, a definite diagnosis of MFS was verified. Information regarding the symptomatic enlargement of the four heart chambers and the global hypokinesis remains to be elucidated, and a heart of the reported size in a patient with MFS is rare. However, it may be due to the severe mitral regurgitation leading to increased left atrium and left ventricular preload, associated with left atrium and left ventricular dilation. Without the severe tricuspid regurgitation or elevated pulmonary arterial pressure, it remains unknown as to how dilation in the right atrium and right ventricle occurred. The observed valvular insufficiencies in the mitral valve do not typically result in such a severe dilation of the four cardiac chambers. Notably, the left ventricular end diastolic dimension was $9.83 \mathrm{~cm}$, which is a rare occurrence, even in dilated cardiomyopathy and to the best of our knowledge, had not been previously reported in patients with MFS. The mutation in exon 27 (c.3386G>A) was previously characterized in an MFS patient with mitral valve prolapse and aortic root dilation (8). Additionally, mutations in exon 52 may result in mitral valve prolapse or aortic dilatation in patients with MFS (14). However, in the present case, the absence of mitral valve prolapse or aortic dilation, suggested that mutations in exon 27 (c.3442C $>$ G) and exon 52 (c.6388G $>$ A) may be associated with the aforementioned atypical cardiovascular manifestations, including the abnormally sized heart. The present study hypothesized that the severe cardiac chamber dilatation was a primary physical sign of MFS and missense mutations in FBN-1 contributed to these pathomorphological alterations. A wide range of MFS-associated mutations have been characterized to date, however a specific genotype/phenotype associated pattern remains to be elucidated. The presence of an FBN-1 mutation does not predict the severity of the MFS phenotype, however it contributes to the increasing demand for genetic analysis of various diseases, and may aid in the research for the understanding of the pathogenesis of MFS.

In conclusion, early medical and surgical management may improve the life expectancy of patients and advanced research holds the promise of further improvements. This case illustrates the importance of obtaining a complete family history, and the value of clinical correlation while assessing patients with unusual physical findings. Early diagnosis of this disease by physicians will help in initiating treatment and appropriate specialist management and furthermore, patient education and genetic counselling will result in early and complete diagnosis. The present report added novel mutations to the genotype-phenotype spectrum regarding the FBN-1 association with MFS. This further emphasizes the importance of an exhaustive clinical investigation to evaluate the necessity of performing the molecular analysis.

\section{References}

1. Pearson GD, Devereux R, Loeys B, Maslen C, Milewicz D, Pyeritz R, Ramirez F, Rifkin D, Sakai L, Svensson L, et al: Report of the national heart, lung, and blood institute and national Marfan foundation working group on research in Marfan syndrome and related disorders. Circulation 118: 785-791, 2008.

2. Judge DP and Dietz HC: Marfan's syndrome. Lancet 366: 1965-1976, 2005

3. Collod-Béroud G, Le Bourdelles S, Ades L, Ala-Kokko L, Booms P, Boxer M, Child A, Comeglio P, De Paepe A, Hyland JC, et al: Update of the UMD-FBN1 mutation database and creation of an FBN1 polymorphism database. Hum Mutat 22: 199-208, 2003.

4. Meng B, Li H, Yang T, Huang S, Sun X and Yuan H: Identification of a novel FBN1 gene mutation in a Chinese family with Marfan syndrome. Mol Vis 17: 2421-2427, 2011.

5. Ammash NM, Sundt TM and Connolly HM: Marfan syndrome-diagnosis and management. Curr Probl Cardiol 33: 7-39, 2008

6. Dormand $\mathrm{H}$ and Mohiaddin RH: Cardiovascular magnetic resonance in Marfan syndrome. J Cardiovasc Magn Reson 15: 33, 2013.

7. Dong J, Bu J, Du W, Li Y, Jia Y, Li J, Meng X, Yuan M, Peng X, Zhou A and Wang L: A new novel mutation in FBN1 causes autosomal dominant Marfan syndrome in a Chinese family. Mol Vis 18: 81-86, 2012.

8. El-Aleem AA, Karck M, Haverich A, Schmidtke J and Arslan-Kirchner M: Identification of 9 novel FBN1 mutations in German patients with Marfan syndrome. Hum Mutat 14: 181, 1999.

9. De Paepe A, Devereux RB, Dietz HC, Hennekam RC and Pyeritz RE: Revised diagnostic criteria for the Marfan syndrome. Am J Med Genet 62: 417-426, 1996.

10. Nijbroek G, Sood S, Mclntosh I, Francomano CA, Bull E, Pereira L, Ramirez F, Pyeritz RE and Dietz HC: Fifteen novel FBN1 mutations causing Marfan syndrome detected by heteroduplex analysis of genomic amplicons. Am J Hum Genet 57: 8-21, 1995.

11. Morse RP, Rockenmacher S, Pyeritz RE, Sanders SP, Bieber FR, Lin A, MacLeod P, Hall B and Graham JM Jr: Diagnosis and management of infantile marfan syndrome. Pediatrics 86: 888-895, 1990.

12. Brown OR, DeMots H, Kloster FE, Roberts A, Menashe VD and Beals RK: Aortic root dilatation and mitral valve prolapse in Marfan's syndrome: An ECHOCARDIOgraphic study. Circulation 52: 651-657, 1975.

13. Sisk HE, Zahka KG and Pyeritz RE: The Marfan syndrome in early childhood: Analysis of 15 patients diagnosed at less than 4 years of age. Am J Cardiol 52: 353-358, 1983.

14. Comeglio P, Johnson P, Arno G, Brice G, Evans A, Aragon-Martin J, da Silva FP, Kiotsekoglou A and Child A: The importance of mutation detection in Marfan syndrome and Marfan-related disorders: Report of 193 FBN1 mutations. Hum Mutat 28: 928, 2007.

15. Chung BH, Lam ST, Tong TM, Li SY, Lun KS, Chan DH, Fok SF, Or JS, SmithDK, Yang W and Lau YL: Identification of novel FBN1 and TGFBR2 mutations in 65 probands with Marfan syndrome or Marfan-like phenotypes. Am J Med Genet A 149A: 1452-1459, 2009. International (CC BY-NC-ND 4.0) License. 